\title{
TV/Series
}

11 | 2017

Philosopher avec Battlestar Galactica

\section{A Tale of Two Toasters : Évolutions de la figure du Cylon dans Battlestar Galactica entre la version de Glen Larson (ABC, 1978-1979) et le reboot de Ronald D. Moore (Syfy, 2003-2009)}

Donna Spalding Andréolle

\section{(2) OpenEdition}

\section{Journals}

Édition électronique

URL : http://journals.openedition.org/tvseries/2065

DOI : $10.4000 /$ tvseries.2065

ISSN : 2266-0909

Éditeur

GRIC - Groupe de recherche Identités et Cultures

Référence électronique

Donna Spalding Andréolle, «A Tale of Two Toasters : Évolutions de la figure du Cylon dans Battlestar Galactica entre la version de Glen Larson (ABC, 1978-1979) et le reboot de Ronald D. Moore (Syfy, 2003-2009) », TV/Series [En ligne], 11 | 2017, mis en ligne le 11 juin 2017, consulté le 05 mai 2019 URL : http://journals.openedition.org/tvseries/2065; DOI : 10.4000/tvseries.2065

Ce document a été généré automatiquement le 5 mai 2019.

TV/Series est mis à disposition selon les termes de la licence Creative Commons Attribution - Pas d'Utilisation Commerciale - Pas de Modification 4.0 International. 


\title{
A Tale of Two Toasters : Évolutions de la figure du Cylon dans Battlestar Galactica entre la version de Glen Larson (ABC, 1978-1979) et le reboot de Ronald D. Moore (Syfy, 2003-2009)
}

\author{
Donna Spalding Andréolle
}

Au cœur de la saga Battlestar Galactica - et en particulier dans sa version revisitée par Ronald D. Moore - se situe la figure du Cylon, être artificiel créé par l'homme mais dont l'évolution ne se réalise pleinement qu'après une rébellion contre l'espèce humaine. Alors que dans la série d'origine de Glen Larson (ABC, 1978-1979) les Cylons se limitent essentiellement à une armée de robots commandée par le traître Baltar (pour le compte d'un mystérieux leader Cylon appelé « Dictateur Suprême »), il est possible d'y discerner déjà un questionnement sur la frontière entre la machine et l'homme. On note par exemple la spécificité du Cylon Lucifer, capable d'ironie dans ses échanges avec Baltar, ou la hiérarchie militaire des Cylons, qui indique une différentiation dans le niveau de conscience et d'individualisation de ces êtres artificiels. C'est dans l'épisode double intitulé The Gun on Ice Planet Zero (S01E08-09, diffusé les 22 et le 29 octobre 1978) que l'on aperçoit les premières réflexions de fond sur le sens donné à l'humanité dans la série. Cet épisode marque d'ailleurs la fin d'une présence forte des Cylons, qui ne réapparaissent que brièvement dans l'épisode de clôture. Ces questionnements, en revanche, sont au cœur même du développement de la figure du Cylon dans le remake de Ron D. Moore : la transformation du Cylon en une créature physiquement ressemblante à l'homme, programmée dès lors à se comporter en humain, pose de réels problèmes tant aux colons en guerre qu'aux Cylons eux-mêmes. Affranchis de l'emprise humaine par l'apocalypse 
intergalactique qu'ils ont déclenchée, les Cylons sont néanmoins prisonniers de leur propre système de pensée collective dictée par l'Hybride ; et c'est au contact des humains, souvent au sein de relations amoureuses (les couples Baltar-Caprica Six et Helo-Athena parmi d'autres) que les Cylons prennent conscience de leur individualité et de leur librearbitre. Au-delà des considérations philosophiques sur la nature de ces êtres et des pistes de réflexion qu'ils offrent pour une réactualisation de certains débats sur l'humain, le posthumain ou le transhumain, nous proposerons ici d'analyser les mises en relation entre les visions de l'altérité proposées dans les deux séries et les évolutions culturelles du genre science-fictionnel américain dans des contextes sociopolitiques très différents.

\section{Altérité et science-fiction américaine}

2 Les deux versions de Battlestar Galactica nous présentent des grandes traditions de la science-fiction dans sa spécificité américaine. Tout d'abord, la science fiction américaine puise ses sources dans le western et prolonge de ce fait la mythologie nationale de la Frontière et l'idéologie attenante. Les conditions particulières de la publication du genre à la fin du XIX siècle - fiction bon marché vendue sous forme de dime novels ${ }^{1}$-, font de la science-fiction américaine une littérature de la culture populaire, ce qui la différencie fondamentalement de la science-fiction britannique ou de la science-fiction française. Là où la science-fiction britannique, à l'instar des œuvres d'H. G. Wells par exemple, traite, dans une approche philosophique, des problèmes liés au futur de l'Homme, la sciencefiction américaine, à l'instar des œuvres d'Edgar Rice Burroughs (remis au goût du jour par le film John Carter of Mars'), traite du périple du héros national, garant de la propagation de la démocratie dans les espaces sauvages de la wilderness. La proximité thématique du western et de la science-fiction, d'abord dans les dime novels, ensuite dans les productions cinématographiques dès les premiers films muets, et enfin dans les séries télévisées, donne naissance au sous-genre du space opera (expression inspirée du horse opera ${ }^{3}$ ). Le scénario du space opera exalte l'esprit pionnier et la technologie utilisée pour propager la "civilisation" (dans son acception occidentale) aux confins de l'univers; l'espace devient la dernière frontière de la lutte entre les forces du Bien et du Mal. Battlestar Galactica, série contemporaine de la Guerre des Étoiles (1977) de George Lucas, illustre clairement les liens forts entre ces deux genres, comme en témoignent ici ces photos des héros respectifs, Han Solo (Harrison Ford) et Starbuck (Dirk Benedict). 
Fig. 1 : Han Solo et Starbuck dans la version 1978
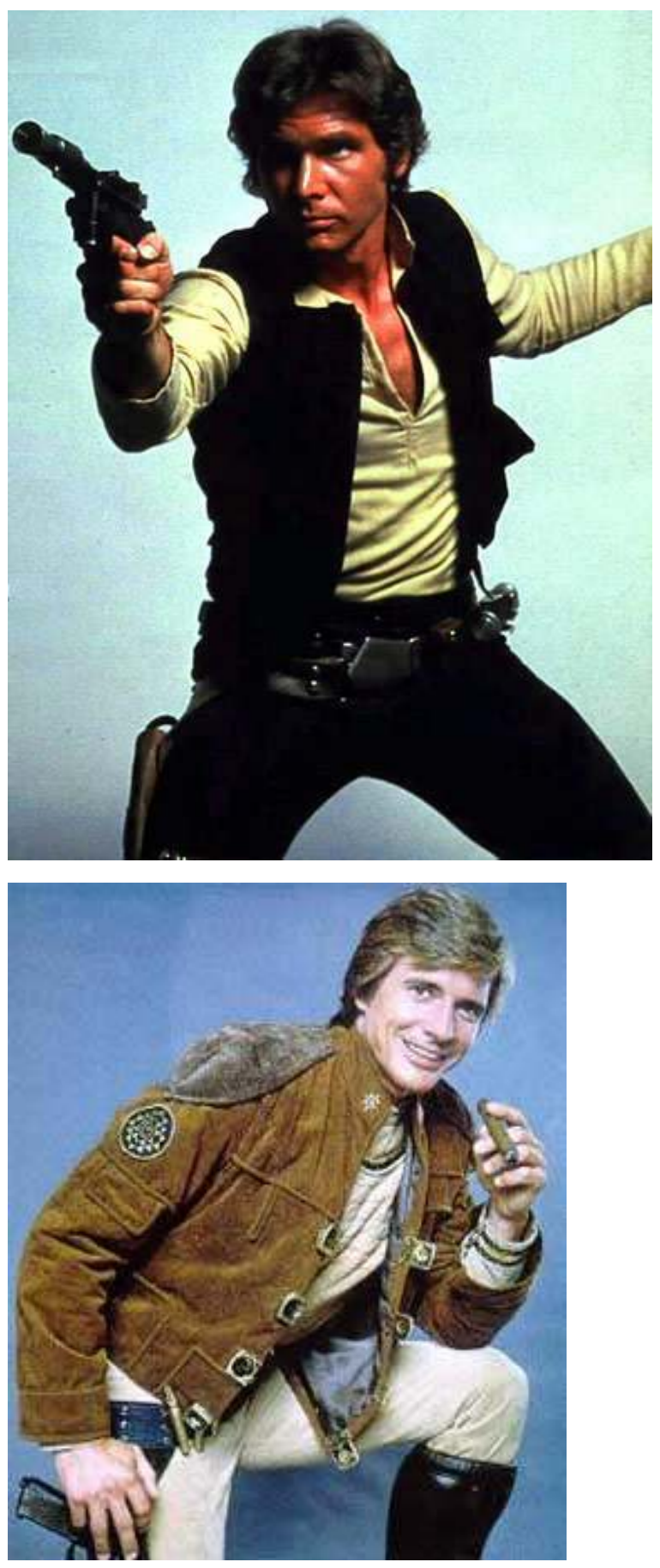

3 Larson semble, dans son écriture de la série source, prisonnier des schémas de la Guerre des Étoiles, succès planétaire de 1977 : par exemple, l'épisode intitulé « The Lost Warrior » (S01E06) inclut une scène de duel dans un saloon sur une planète désertique qui rappelle fortement la scène célèbre sur Tatooine, tout en faisant écho à un épisode de Star Trek (1966-1969), " The Spectre of the Gun » (S03E06), dans lequel Kirk et ses officiers doivent revivre la fusillade d'O.K. Corral ${ }^{4}$.

De même, les Cylons, dans la version de 1978, sont majoritairement des soldats-robots sans conscience ni intelligence, fort ressemblants, physiquement, à «Dark Vador » 
(Darth Vader) et à l'armée des clones de la Guerre des Étoiles ${ }^{5}$. Ici le traitement de l'altérité puise dans les traditions de la littérature de la Frontière, dans une réitération des relations avec l'Autre racial et culturel de l'expérience américaine qui remonte aux récits de captivité puritains du XVII ${ }^{\mathrm{e}}$ siècle. Comme l'historien culturel américain Richard Slotkin nous le rappelle dans Regeneration Through Violence: The Mythology of the American Frontier 1600-1860:

The cultural anxieties and aspirations of the colonists found their most dramatic and symbolic portrayal in the accounts of the Indian wars. The Indian war was a uniquely American experience [in which] they could suggest their own superiority to the home English by exalting their heroism in battle, the peculiar danger of their circumstances, and the holy zeal for English Christian expansion [...]. It was within this genre of colonial Puritan writing that the first American mythology took shape - a mythology in which the hero was the captive or victim of devilish American savages and in which his (or her) heroic quest was for religious conversion and salvation ${ }^{6}$.

L'Indien (compris en tant que construction occidentale), incarne la confrontation culturelle dans laquelle deux visions du monde s'opposent par un regard «en miroir », dans lequel chacune des deux cultures perçoit et juge l'autre. L'Indien concentre l'ambivalence ressentie par le colon blanc, dans le fantasme du risque de contamination que le contact avec le "sauvage " entraînerait pour la "civilisation " blanche. Dans le space opera de la première moitié du $\mathrm{xx}^{\mathrm{e}}$ siècle, l'Alien (extra-terrestre) remplace l'Indien comme être antithétique de la civilisation dans la vision occidentale du monde, et sert de faire-valoir à la société moderne et/ou au monde de référence du lecteur ou spectateur, même si cette fonction connaîtra une inversion marquante, due à l'impact, à compter des années 1960, des mouvements d'historiographie dite " radicale » (c'est-à-dire d'inspiration marxiste $)^{7}$. Après la Seconde Guerre mondiale et le début de la guerre froide ${ }^{8}$, la peur du communisme a fait de l'invasion un thème de prédilection dans la science-fiction américaine ; cependant ces scenarios d'invasion ne s'attardaient pas sur la complexité de l'altérité, et n'exprimaient pas le désir de contact ou de compréhension puisque le seul souci était de préserver le monde tel qu'il existait (et de préférence sans l'Autre). La version de Battlestar Galactica créée par Glen Larson provient de ce contexte de la guerre froide, et le traitement des Cylons s'en ressent; la série «ré-imaginée » par Ronald D. Moore vingt-cinq ans plus tard, en revanche, arrive à s'affranchir clairement de ces schémas traditionnels pour poser des questions philosophiques sur l'altérité plus proches de celles que l'on trouve dans la tradition sci-fi britannique.

\section{Avatar ou némésis ?}

6 La posture idéologique des deux séries sur la question des relations entre humains et Cylons apparait très tôt, dans le premier épisode de chaque version. Dans la version d'origine, c'est cet échange entre le président des douze colonies et Adama :

PRÉSIDENT. I see the party is not a huge success with all of my children.

ADAMA. What awaits us out there is what troubles me.

PRÉSIDENT. But surely you don't cling to your suspicions about the Cylons? They asked for this armistice, they want peace.

AdAma. Forgive me, Mr. President, but they hate us with every fiber of their existence. We love freedom, we love independence - to feel, to question, to resist oppression. To them, it is an alien way of existing they will never accept. 
PRÉSIDENT. But they have, through Baltar. They have sued for peace.

ADAMA. Yes, of course, you are right.

7 La division entre humains et Cylons est claire : d'un côté, une civilisation qui aime la liberté, l'indépendance, et qui résiste à l'oppression; de l'autre, un empire auquel ces concepts sont étrangers. Ce sont des références à peine voilées à la Révolution américaine, et à la terminologie de la doctrine de Truman (1947) qui décrit la vision américaine de la guerre froide. L'axe du Mal est représenté par le mystérieux cerveau de l'invasion Cylon, "Imperious Leader" (le "Suprême Dictateur » dans la traduction française), que l'on voit de dos dans cette première apparition, puis avec la forme humaine ci-dessous dans l'épisode « War of the Gods » (S01E15-16).

Fig. 2 : Le Suprême Dictateur

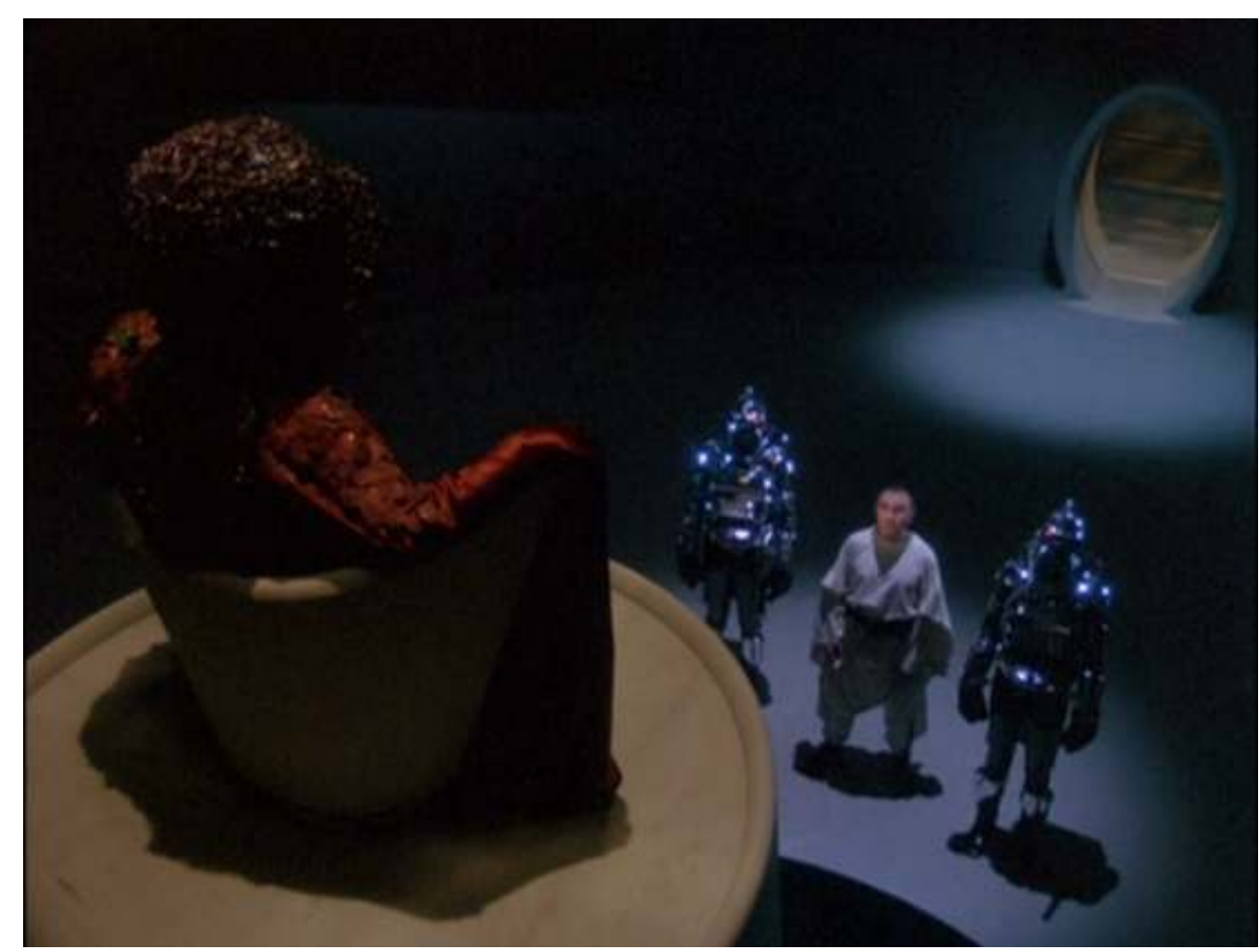




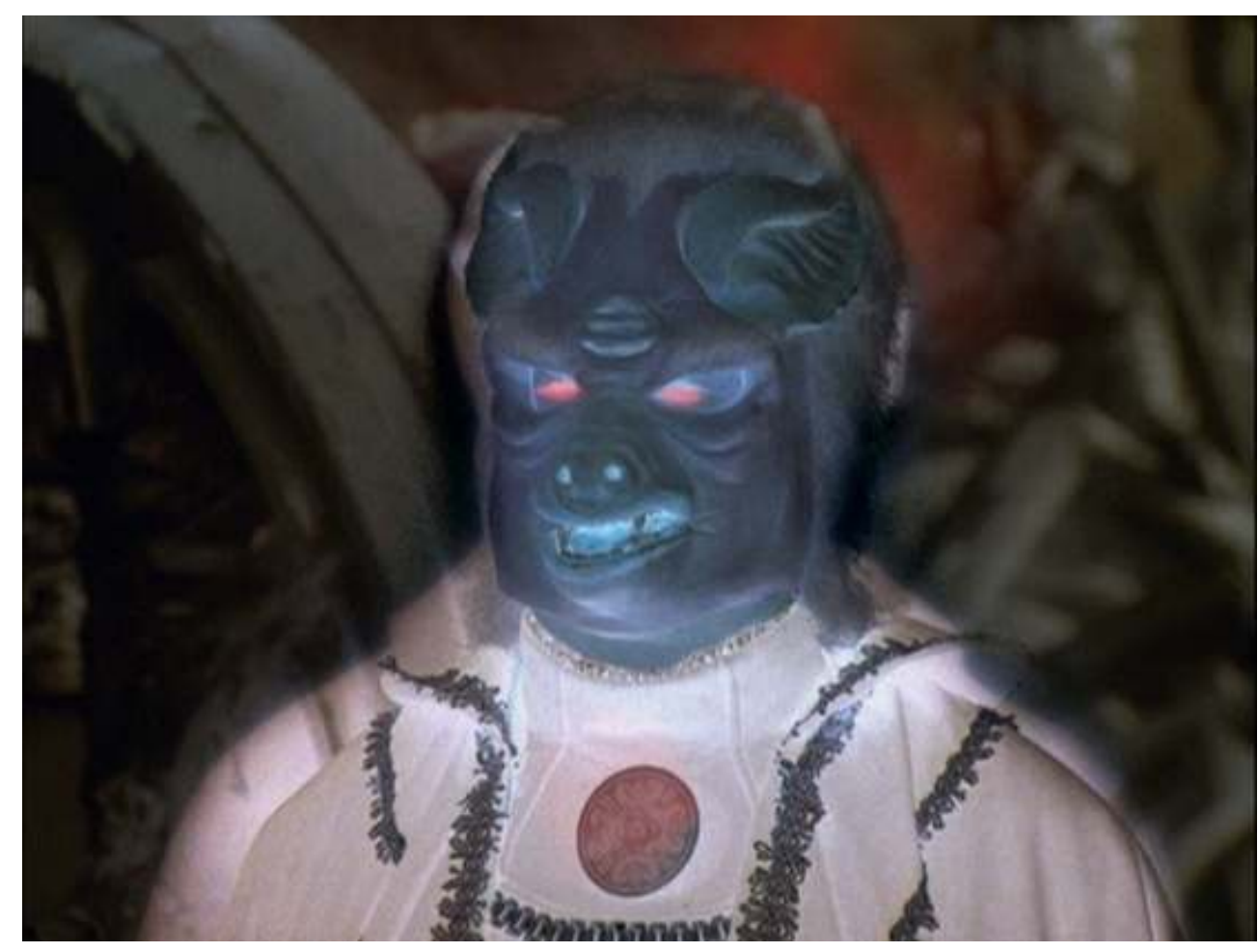

De nombreux autres indices rappellent aux spectateurs le schisme idéologique qui sépare la civilisation de l'Empire du Mal: les tètes des soldats Cylon renvoient à l'image des légionnaires de l'empire romain, voire de l'armée nazie ; Baltar dans les scènes de bataille ressemble à Jules César; le Cylon comparse de Baltar s'appelle Lucifer, et le Suprême Dictateur s'avère avatar de Satan. Enfin, dans l'épisode "Greetings From Earth" (S01E19-20), une scène de lancement de missiles réaffirme la peur d'une guerre nucléaire créée par la crise des missiles de Cuba en octobre 1962, crise immortalisée, dans la culture populaire, par le film Docteur Folamour (Dr. Strangelove, Stanley Kubrick, 1964). 
Fig. 3 : Docteur Folamour (Kubrick, 1964)
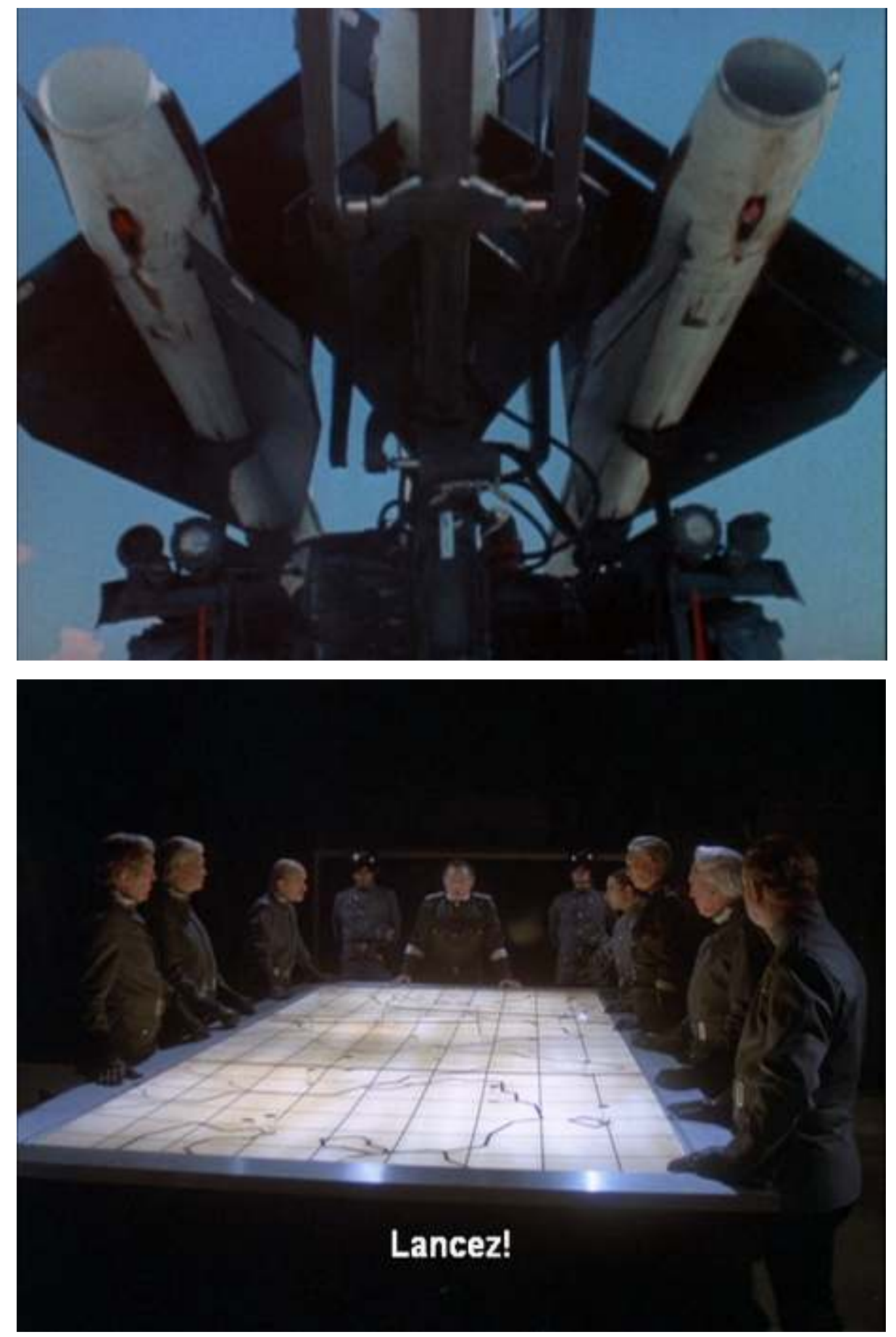

9 Les Cylons imaginés par Larson prennent trois formes distinctes : des soldats-robots, des êtres plus évolués représentés par les personnages de Lucifer et Spectre, et le Suprême Dictateur dont la nature exacte, humaine ou mécanique, demeure un mystère. Il s'agit néanmoins d'aliens stéréotypés dont on nie l'humanité et dont les différences manichéennes sont reconnues et acceptées ; à l'inverse des Cylons dans la version "réimaginée » de Ronald D. Moore, sur le plan technologique, les Cylons de 1978 semblent simplement à égalité avec les humains, et de ce fait moins menaçants. Ils ne provoquent donc pas de peur réelle chez le spectateur, puisque les humains sont capables de les 
battre quelles que soient les conditions dans lesquelles les Cylons les affrontent; malgré leur nombre nettement supérieur (mais moins terrifiant que dans la version 2003 où l'on voit de véritables essaims de raiders autour d'un vaisseau-mère aux proportions monstrueuses), les Cylons de Larson ne peuvent se mesurer à l'intelligence des pilotes de chasse aux tactiques surprenantes car «illogiques» aux yeux de ces créatures artificielles. Par ailleurs, toute dimension effrayante de leur altérité se voit remise en question par des badinages entre Baltar et Lucifer ou encore, dans cette scène de l'épisode "The Young Lords» (S01E11) - les commentaires des soldats-robots et la réplique de Starbuck ne peuvent que faire sourire'.

Fig. 4 : Humour dans la série de Larson

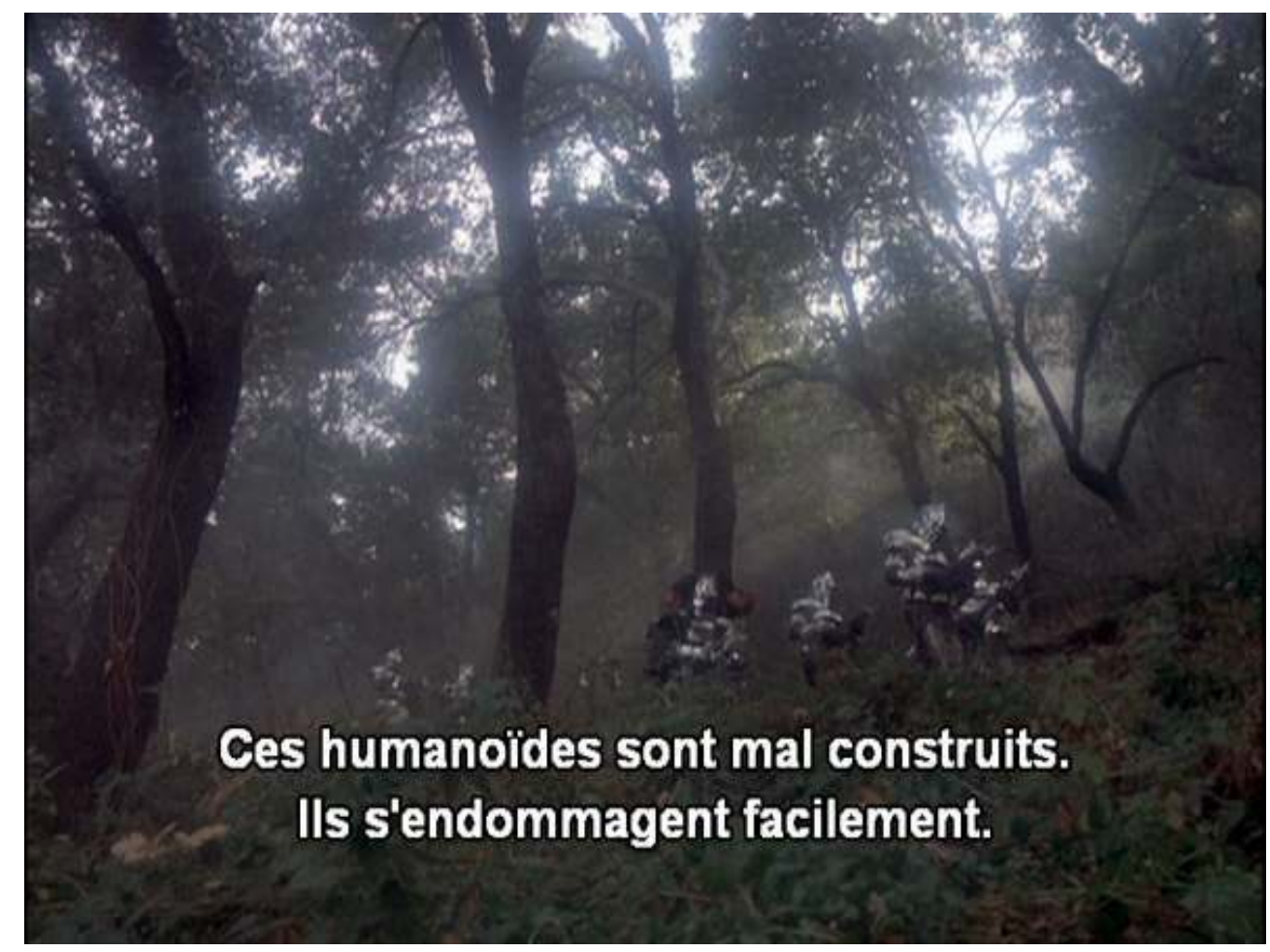




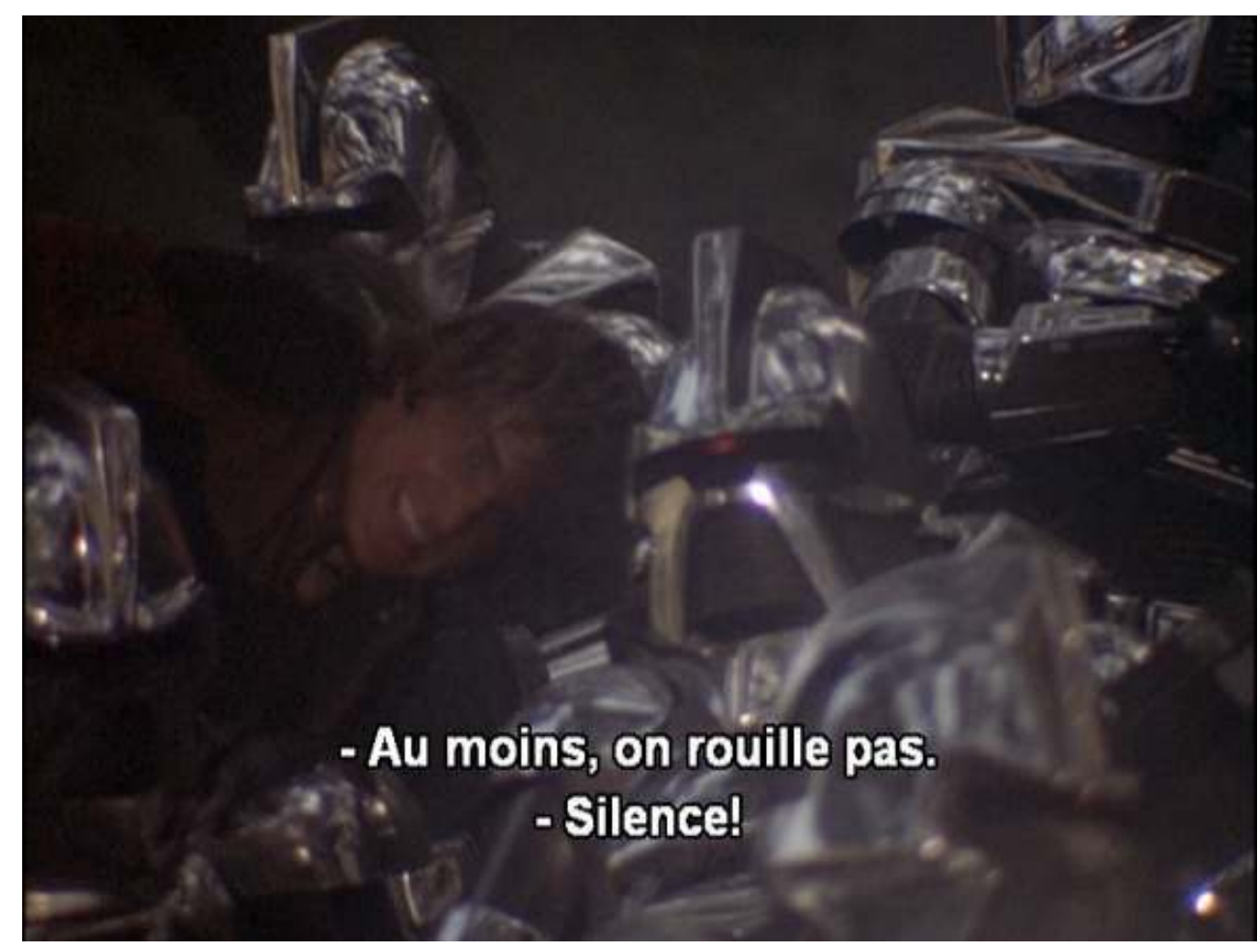

10 Là où les regards croisés entre l'humain et l'Autre servent traditionnellement à renforcer l'altérité, ici l'altérité de chacun est redéfinie de manière délibérément incongrue, d'où l'effet comique mais aussi un effet de «rapprochement». Peut-être pourrait-on interpréter cet humour comme une critique, de la part de Glen Larson, du discours de la peur face aux communistes et aux « autres " plus généralement? D'un autre côté, les victoires systématiques des humains sur les Cylons apparaissent comme une réitération pure et simple des mythes nationaux de la conquête territoriale chers au space opera.

11 Le côté kitsch, voire camp - comme le dit Ronald D. Moore ${ }^{10}$ - de Battlestar Galactica 1978 réside ainsi dans la sacralisation des stéréotypes de la science-fiction américaine au détriment d'un discours idéologique fort, pourtant annoncé dans les premiers instants de la série. Une première explication peut se trouver dans ce que Kingsley Amis expose dans New Maps of Hell (1961) : que la science-fiction met en scène des personnages "types " (voire des stéréotypes) puisque le genre traite de la condition humaine plutôt que de l'individu, donnant lieu à sa formule célèbre "Idea as Hero ». Joanna Russ, auteure féministe américaine de science fiction dans les années 1960-7011 notait que «les protagonistes de la science-fiction sont traités en tant que collectivité et non pas en tant qu'individus, même si certains personnages peuvent être exemplaires ou représentatifs » ${ }^{12}$. Serait donc responsable de la disparition du protagoniste l'intérêt que portent les auteurs à la condition humaine et au fonctionnement de la collectivité, plutôt qu'aux actes de l'individu.

12 Une deuxième explication possible des personnages stéréotypés serait sans doute la mort précoce de la série dans sa seconde saison, mort programmée qui aurait privé Larson et les scénaristes de la possibilité de développer plus longuement les relations étranges entre les humains et leurs ennemis. Peut-être aussi n'était-il pas envisageable de subvertir une rhétorique hégémonique ou de détourner le space opera vers d'autres types de représentation ou de discours; après tout, les tentatives de Gene Roddenberry dans Star Trek (CBS, 1966-1969) avaient échoué ${ }^{13}$, pourtant dans une période de contestation 
propice à de tels détournements. L'Amérique de la fin des années 1970 fut, dans le sillage de Watergate et au milieu de la débâcle des otages de Téhéran, sans doute davantage friande d'histoires à la gloire du héros américain triomphant face à l'adversité la plus insurmontable; et le public de prédilection de la science-fiction dans ses formes traditionnelles reste celui des adolescents. Voulant ainsi capitaliser sur l'engouement pour Star Wars, la chaîne $\mathrm{ABC}$ privilégia donc la production d'une série qui ne cherchait pas à bousculer les habitudes du public par un contenu trop « intellectuel », reproche fait à Gene Roddenberry en son temps - $\mathrm{ABC}$ mit d'ailleurs fin à Star Trek malgré les protestations organisées par les fans ${ }^{14}$.

13 Les choix politiques du Battlestar Galactica de Ronald D. Moore sont tout autres, dans un contexte culturel également bouleversé : on peut lire dans ce reboot une métaphore de la guerre au terrorisme ("War on Terror») et de son impact sur les idéaux politiques, éthiques et moraux de la société américaine de "l'après 11 septembre» (post-9/11) ${ }^{15}$. Malgré les affirmations de Moore et de certains acteurs de la série ré-imaginée selon lesquelles le scénario est resté inchangé par rapport à l'original, la posture idéologique telle qu'elle est exprimée dans la séquence du discours d'Adama après l'attaque des Cylons dans l'épisode pilote démontre une transformation radicale dans la perception des relations entre humains et Cylons :

ADAMA. The Cylon war is long over, yet we must not forget the reasons why so many sacrificed so much in the cause of freedom. The cost of wearing the uniform can be high, but... (long silence)... sometimes it's too high. We thought that when we fought the Cylons, we did it to save ourselves from extinction, but we never answered the question why? Why are we as a people worth saving? We still commit murder because of greed and spite, jealousy, and we still visit all of our sins upon our children. We refuse to accept responsibility for anything we've done, like we did with the Cylons. We decided to play God, create life; when that life turned against us, we comforted ourselves in the knowledge that it really wasn't our fault, not really. You cannot play God then wash your hands of the things that you have created. Sooner or later, the day comes when you can't hide from the things you've done any more.

14 Ce discours opère aux antipodes de celui de la version d'origine: ici Adama offre aux spectateurs une interprétation de la guerre contre les Cylons plus près de la prédication puritaine que du discours du chef militaire cherchant à rallier ses troupes en temps de crise. L'insistance sur les notions de faute et de péché, et l'idée que la guerre avec les Cylons représente une forme de punition divine face à l'hubris humaine font écho aux questionnements et explications des attentats du 11 septembre 2001 dans la presse américaine immédiatement après les attentats ${ }^{16}$. Grâce à la distanciation que permet le genre de l'anticipation, Moore produit, comme il l'explique dans la bible de la série ${ }^{17}$, une version de Battlestar Galactica qui se veut allégorie des États-Unis de 2004-2010 - allégorie d'après lui facilement décodable par les téléspectateurs. C'est spécifiquement dans son traitement de l'altérité que Moore transforme le space opera de Battlestar Galactica en fiction politique, phénomène assez rare dans la tradition science-fictionnelle américaine et réservé aux meilleurs écrivains tels que Ray Bradbury (notamment dans Fahrenheit 451) et certaines auteures féministes telles qu'Ursula K. Le Guin, Suzy McKee Charnas et Octavia E. Butler ${ }^{18}$. Cette altérité des Cylons est placée au centre de l'intrigue dès la séquence du générique (que l'on revoit au début de chaque épisode); ce montage de quelques phrases et quelques images nous avertit des dimensions les plus troublantes et les plus monstrueuses de ces « Autres ». 
Fig. 5 : générique de la série de Ron D. Moore

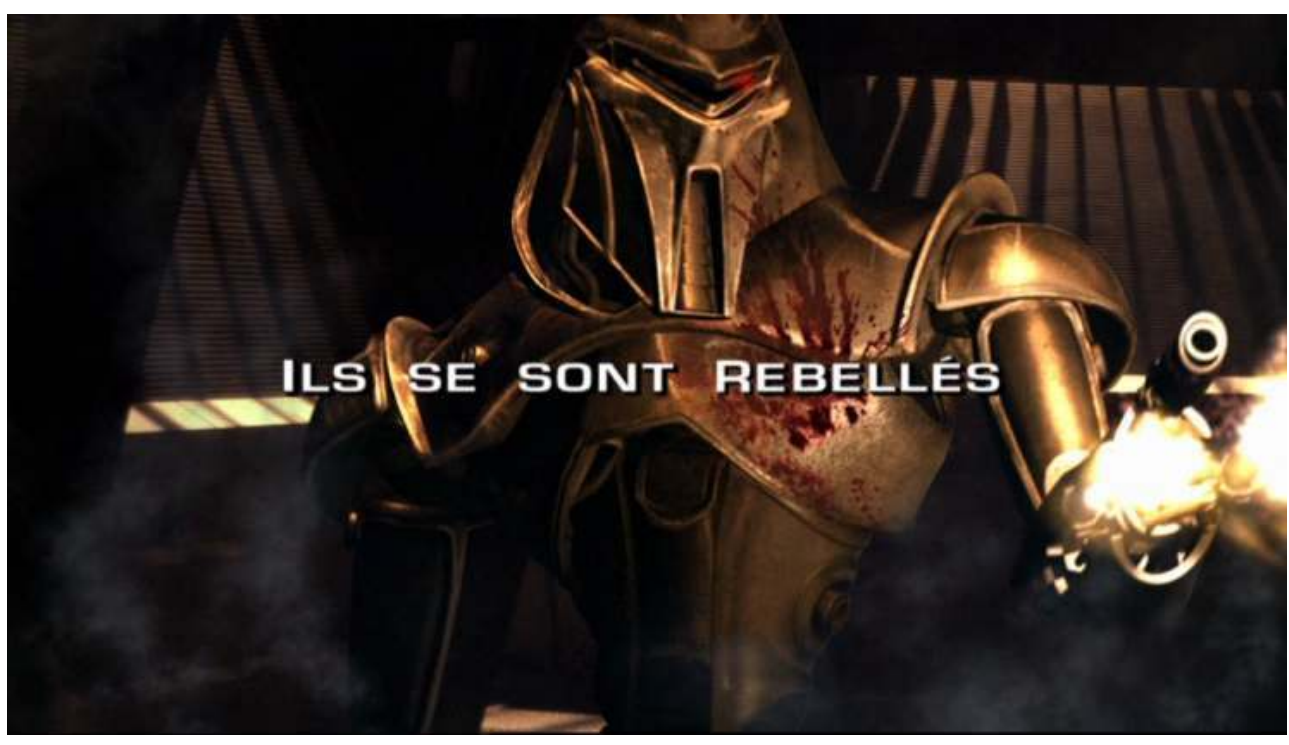

Comme dans Battlestar Galactica 1978, les Cylons prennent trois formes distinctes selon leur niveau d'évolution : les avions de chasse militaires appelés raiders (l'équivalent des vipers chez les humains), les Centurions, véritables machines à tuer de type humanoïde géant, et les Cylons impossibles à distinguer des êtres humains, répliqués en douze modèles - y compris certains qui ne savent pas qu'ils en sont (" sleeper agents»). On découvre leur identité durant les quatre saisons et notamment dans l'épisode final de la saison 3, «Crossroads part $2 »$ (S03E20). Ces trois formes illustrent non seulement l'état technologique supérieur de cette intelligence artificielle par rapport à son créateur humain mais aussi toute l'ambiguïté quant à leur nature exacte, suspendue entre machine et homme. Si les Centurions sont complètement et visiblement mécaniques, et les douze modèles semblent humains à tout égard, les raiders se composent d'une carapace métallique renfermant un être biologique fait de viscères et de sang; cette nature partiellement biologique explique l'intelligence manifeste des raiders lors de leurs engagements contre les pilotes du Galactica.

16 Les Cylons constituent l'ennemi ultime: ils n'hésitent pas à déployer des armes de destruction massive dans leur attaque simultanée de Caprica et des autres planètes de la confédération des colonies; ils disposent d'une armée mécanisée invincible car remplaçable à l'infini ; ils infiltrent les lignes ennemies incognito et disposent de sources supérieures d'information venant de collaborateurs humains; ils envahissent et détruisent les systèmes de défense de l'intérieur par la propagation de virus dans le réseau informatique reliant les vaisseaux de la flotte coloniale. C'est justement une sorte d'ironie cosmique que le seul battlestar ou vaisseau à survivre à l'apocalypse soit le Galactica, le plus ancien, le plus "low tech" avec un commandant aux méthodes rétrogrades. Ainsi pourrait-on dire que les Cylons représentent une combinaison de toutes les craintes américaines depuis la guerre froide: dangers de la technologie nucléaire entre les mains de régimes instables, peur d'un virus qui provoquerait l'apocalypse cybernétique ${ }^{19}$ ), crainte d'attentats terroristes capables d'anéantir des villes entières. Les Cylons sont à la fois avatar et némésis, copies infinies de ce que l'Homme désire être (beau, puissant, surhumain, immortel) et un rappel de la monstruosité née de ces mêmes désirs. On peut voir en Numéro Six (Caprica Six) une incarnation de la déesse 
Némésis car c'est elle qui annonce l'apocalypse à Baltar, et qui sera la porte-parole des raisons de la vengeance des Cylons contre leurs créateurs. Au début de l'épisode « Mission Suicide » (S03E01) la voix off de Laura Roslin nous expose la futilité de la résistance des humains de New Caprica sous l'occupation des Cylons: "Tuer les Cylons n'est pas suffisant parce qu'ils ne meurent pas. Ils ressuscitent et reviennent parmi nous. C'est terrifiant ${ }^{20}$. » Ainsi le désir d'immortalité, source de fascination, est-il également source de terreur et de révulsion; couplés avec l'incertitude sur la nature même de cet Autre, ces sentiments simultanés d'attraction et de rejet font des Cylons de Ronald D. Moore des figures complexes qui ébranlent des attitudes culturelles sur l'altérité et les relations entre les États-Unis et le monde, sous la forme indirecte de l'allusion.

\section{Une monstruosité inversée}

17 Ronald Moore pousse encore plus loin ses réflexions sur les différences entre humanité et altérité, toujours dans le premier épisode de la saison 3 («Mission suicide »), moment clé du développement de cette problématique. Dans ce dialogue, les principaux Cylons débattent de la meilleure manière de combattre l'insurrection des colons de New Caprica :

CAVIL 1. Let's review why we are here, shall we? We're supposed to bring the word of "God" to the people, right?

CAVIL 2. To save humanity from damnation by bringing the love of "God" to these poor benighted people.

NUMBER 6. We're here because a majority of the Cylon [sic] felt that the slaughter of mankind had been a mistake.

NUMBER 8. We're here to find a new way to live in peace, as God wants us to live.

CAVIL 2. And it's been a fun ride so far. But I want to clarify our objectives: if we're bringing the word of God, then it follows that we should employ any means to do so, any means.

CAVIL 1. Yes, fear is the key article of faith, as I understand, so perhaps it is time to instill a little more fear in people's hearts and minds. Let's, ah, let's execute Baltar. BALTAR. What? WHAT?!

NUMBER 6. That's not going to happen.

18 Cette monstruosité faite d'actes meurtriers et d'hypocrisie ne sont pas du seul fait des Cylons : d'abord le discours des avatars de Cavil dans cette scène résume étrangement la politique de l'administration Bush dans la guerre contre le terrorisme. Comme le démontrait si bien Michael Moore dans son film Fahrenheit 9/11 (2004), un des objectifs cachés de la guerre contre la terreur - Bush ayant choisi l'expression «War on Terror » plutôt que "war on terrorism » - était de maintenir la population américaine dans un état permanent de peur. Le cynisme de Cavil et son évocation de la volonté de «Dieu » (qu'il met entre guillemets, qu'il mime d'un geste des doigts) pour instaurer la peur chez les colons-résistants de New Caprica constituent une rhétorique qui est familière au spectateur américain, rendant encore plus floue la ligne de démarcation entre humains et Cylons, d'autant plus que les objections des avatars des Numéros Six et Huit nous rapprochent au moins des quelques Cylons à la recherche d'une cohabitation paisible avec les humains. Hélas, le contact prolongé entre Cylons et humains semble mutuellement néfaste: tandis que les Cylons-collaborateurs (Caprica Six, Boomer et Leoben en particulier) s'élèvent finalement contre le génocide de l'humanité, les humains, eux, deviennent les meurtriers monstrueux de leur ennemi. Toujours dans l'épisode "Mission suicide", le spectateur assiste au meurtre sauvage de Leoben par Starbuck. Pire, Loeben lui fait remarquer, lorsqu'il réapparaît quelques scènes plus tard, 
que cela fait la cinquième fois dans la même journée qu'elle agit ainsi. Ce meurtre est filmé de manière à suggérer que Kara est folle, car elle passe du meurtre à son repas d'un air insouciant, la main encore ensanglantée par les coups de poignard dont elle a criblé sa victime. De même, lorsque l'humain choisi par la résistance pour commettre un attentat suicide contre Baltar lors d'une cérémonie officielle se regarde dans la glace une dernière fois, il nous renvoie une image fragmentée et monstrueuse.

Fig. 6 : Image fragmentée et monstrueuse de l'humain

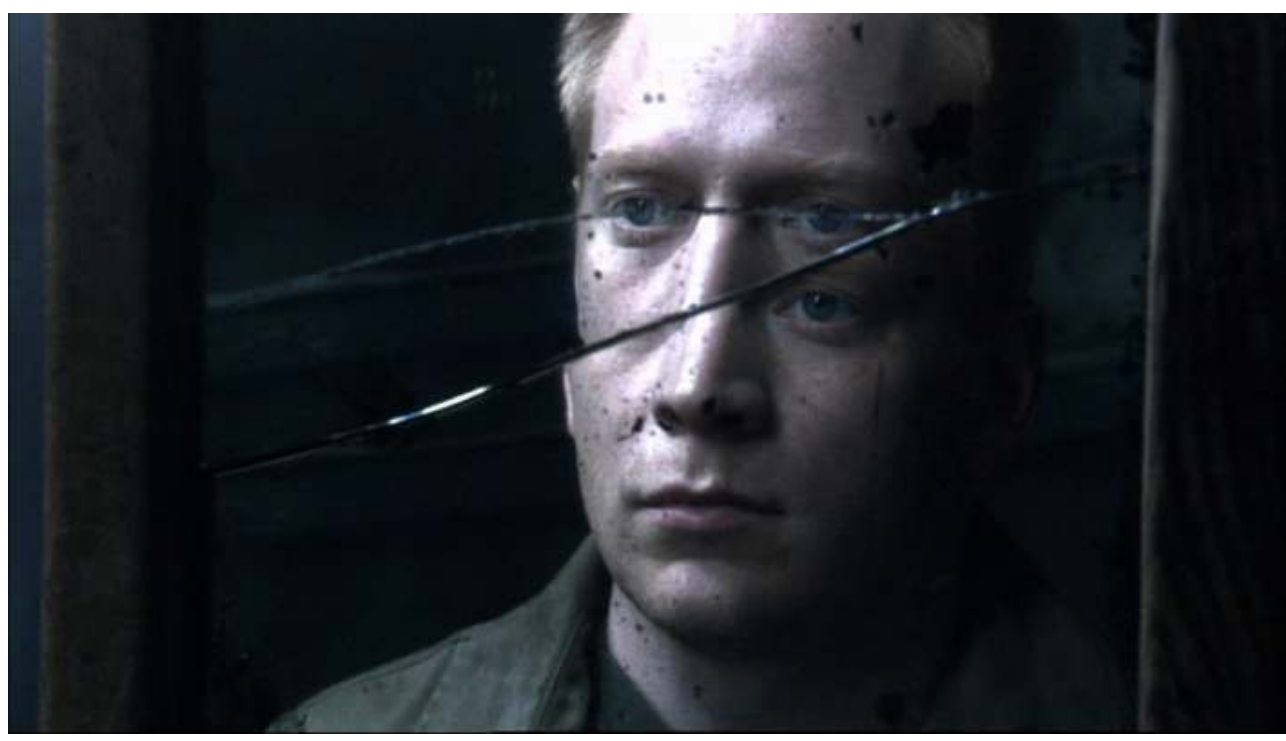

Il semblerait que le contact prolongé entre les colons et leurs créations posthumaines ne puisse mener qu'à une perte d'identité et une évolution incertaine. On peut alors se poser la question du sens à donner à l'allégorie plutôt sombre de Moore. Je postulerai qu'une lecture double de cette monstruosité inversée se présente à nous : soit on est encore dans les schémas de la littérature de Frontière où le colon doit régresser à l'état sauvage avant de pouvoir émerger comme nouvel Adam dans la terre promise du Nouveau Monde auquel cas nous pouvons dire que la série est conservatrice et reste sagement dans les limites de la science-fiction américaine traditionnelle -, soit on estime que Moore cherche à démontrer que la monstruosité est une question de regard et que cette monstruosité est aussi en nous. Dans ce cas, l'on comprend que l'altérité n'est que prétexte à commettre des actes monstrueux, et que la série est progressiste, puisqu'elle nous invite, certes, à une introspection personnelle, mais également sociétale.

Pour conclure, en comparant l'évolution de la figure du Cylon, d'un Autre clairement défini dans la version d'origine de Battlestar Galactica, à l'altérité ambigüe dans la série réimaginée par Ronald D. Moore, il est évident que la raison principale des différences dans l'approche esthétique et idéologique de l'Autre réside en grande partie entre textes et contextes respectifs : guerre froide pour l'une, «guerre contre la terreur " pour l'autre. Le traumatisme du 11 septembre 2001 impacte le concept d'altérité, rendu flou dans la version de Moore par la ressemblance physique entre humains et Cylons les plus évolués, et par l'inversion des croyances religieuses entre monothéistes et polythéistes qui bouleverse les oppositions habituelles de la SF américaine, plaçant ainsi Battlestar Galactica dans le genre de la fiction politique d'anticipation pour petit écran. La version du XXI siècle se place sans ambiguïté dans un ensemble de séries de «l'après 11 septembre 
(2001) $»^{21}$ mettant en lumière l'impact d'un traumatisme national sur la manière de voir le monde, tout en proposant un autre avenir possible que la « guerre sans fin ».

\section{BIBLIOGRAPHIE}

BATAILLE Sylvaine, "Les pièges du temps: la réappropriation de l'Antiquité gréco-latine dans Battlestar Galactica (Sci-Fi, 2003-2009)”, Les pièges des nouvelles séries télévisées américaines: mécanismes narratifs et idéologiques, éd. Sarah Hatchuel et Monica Michlin, GRAAT online \# 6, déc. 2009, p. 86-106. http://www.graat.fr/backissuepiegesseriestv.htm

CARroll Peter N. et David NoBle, The Free and the Unfree: A New History of the United States, New York, Penguin Books, 1988 (deuxième édition).

GENOVESE Eugene D., The Political Economy of Slavery: Studies in the Economy \& Society of the Slave South, Middletown, Connecticut, Wesleyan University Press, 1961.

KAVENEY Roz et Jennifer stoy (éd.), Battlestar Galactica : Investigating Flesh, Spirit and Steel, Londres, I. B. Tauris, 2010.

MAGUIRE Lori, «"Why Are We as a People Worth Saving?” Battlestar Galactica and the Global War on Terror ", TV/Series $n^{\circ} 1$ : Les Séries télévisées américaines contemporaines : entre la fiction, les faits, et le réel, éd. Ariane Hudelet et Sophie Vasset, 2012, http://tvseries.revues.org/1519

RUSS Joanna, «Towards an Aesthetic of Science Fiction », Science Fiction Studies \#6 Vol 2.2, July 1975. http://www.depauw.edu/sfs/backissues/6/russ6art.htm

SLOTKIN Richard, Regeneration Through Violence: The Mythology of the American Frontier 1600-1860, New York, Harper Perennial, 1973.

SPALDING ANDRÉolLE Donna et Anne Crémieux (éd.), TV/Series n5 5 : Religions en séries, 2014, http:// tvseries.revues.org/435

SPALDING ANDRÉOLLE Donna, Dialectique idéologique et sociale dans la science-fiction féminine aux ÉtatsUnis, 1966-1996: Querelles du présent, utopies du futur, Villeneuve d'Ascq, Presses Universitaires du Septentrion, 1999.

SPALDING ANDRÉOlLE Donna, “ "Beam Me Up Scotty, There's No Intelligent Life Down Here”: The failed (counter)cultural message of Star Trek the Original Series (1966-1969) », EOLLE n 1 : The Woodstock Years, éd. Elizabeth Boucé, 2011, p. 39-46, https://gric.univ-lehavre.fr/spip.php? article124

SPALDING ANDRÉOLLE Donna, « Echoes of the "War on Terror" and Post 9-11 Culture in Battlestar Galactica (Syfy Channel, 2003-2009) », TV/Series n 4 : Écho et reprise dans les séries télévisées II : Représentations - enjeux socioculturels, politiques et idéologiques de la reprise, éd. Sylvaine Bataille et Florence Cabaret, 2013, http://tvseries.revues.org/744.

ZINN Howard, The Southern Mystique, New York, Alfred Knopf, 1964. 


\section{NOTES}

1. Appelés « dime novels » car ils coutaient 10 cents ; il s'agit d'une littérature imprimée sur du papier de très mauvaise qualité et donc peu chère, à destination de lecteurs peu fortunés.

2. John Carter of Mars (réal. Andrew Stanton, 2010). Film inspiré du premier roman de sciencefiction américaine, A Princess of Mars d'Edgar Rice Burroughs (1917), plus connu pour son roman Tarzan of the Apes (1912).

3. Carroll et Noble, The Free and the Unfree, New York, Penguin, 1988 ( $2^{\mathrm{e}}$ éd.), p. 379-380. Cette expression se réfère aux westerns télévisés à partir de 1948 comme The Lone Ranger ( $\mathrm{ABC}$, 1949-1957) ou Hopalong Cassidy (NBC, 1952-1954). Le genre perdure jusque dans les années 1960 avec les séries « western » les plus longues de l'histoire de la télévision américaine, Bonanza (NBC, 1959-1973) et Gunsmoke (CBS, 1955-1975).

4. Pour une lecture de cet épisode de Star Trek, voir Donna Spalding Andréolle, «'Beam me up Scotty, there's no intelligent life down here': The failed (counter)cultural message of Star Trek the Original Series (1966-1969)» dans EOLLE $\mathrm{n}^{\circ} 1$ The Woodstock Years, U. Le Havre, 2011. https:// gric.univ-lehavre.fr/spip.php?article124

5. Glen Larson fut surnommé «Glen Larceny » («Glen le Larcin») par l'auteur de science fiction Harlan Ellison, car de nombreuses séries écrites par Larson, dont Battlestar Galactica, s'inspiraient de blockbusters hollywoodiens.

Pour la citation complète et la liste des exemples voir http://en.wikipedia.org/wiki/ Glen_A._Larson, «Criticism ».

6. Richard Slotkin, Regeneration Through Violence: The Mythology of the American Frontier 1600-1860, New York, Harper Perennial, 1973, p. 21. («C'est dans les récits des guerres indiennes que les angoisses et les aspirations culturelles des colons trouvèrent leur manifestation la plus symbolique et la plus spectaculaire. La guerre contre les Indiens constitua une expérience unique leur permettant d'affirmer leur propre supériorité par rapport aux Anglais de la métropole en exaltant leur héroïsme au combat, les dangers auxquels leur situation les exposait et le zèle qu'ils déployaient pour répandre une culture anglaise et chrétienne. C'est dans ce type de récits puritains qu'une première mythologie américaine vit le jour - une mythologie dans laquelle le héros ou l'héroïne était le prisonnier ou la victime de sauvages américains diaboliques et dont la quête héroïque avait pour but la conversion religieuse et le salut »). Mes remerciements à Ronan Ludot-Vlasak pour cette traduction.

7. Notamment Howard Zinn et Eugene Genovese, tous deux spécialistes d'abord de l'histoire du Sud (respectivement The Southern Mystique, 1962 et The Political Economy of Slavery, 1968) puis figures de proue de l'école américaine d'historiographie d'inspiration marxiste qui cherchait à inverser la perspective historique de l'école d'historiographie dite "consensuelle », dominante jusqu'à la fin des années 1950. Dans la science-fiction, cette inversion de la valeur intrinsèque de l'alien est encore flagrante dans des films tels que Avatar de James Cameron (2010) et John Carter of Mars mentionné plus haut.

8. Mise en place officiellement par le document NSC 68 sous la présidence Truman en mars 1947.

9. CYLON CENTURION. THESE HUMANOIDS ARE NOT WELL CONSTRUCTED. THEY DAMAGE EASILY.

STARBUCK. AT LEAST WE DON'T RUST!

10. Interview de Ronald D. Moore intitulé « De 1978 à nos jours », bonus du DVD du pilote de Battlestar Galactica (2003). La citation exacte est : «Here was Battlestar Galactica, which if nothing else, was one of the campiest and one of the biggest, glossiest over-the-top productions that the seventies produced. After Star Wars came out it was like "let's get one of those," says ABC TV and they did Battlestar Galactica ». 
11. Citons en particulier Picnic on Paradise (1968), And Chaos Died (1970) The Female Man (1975), We Who Are About To... (1977) et The Two of Them (1978).

12. «The protagonists of science fiction are always collective, never individual persons (although individuals often appear as exemplary or representative figures) », Joanna Russ, " Towards an Aesthetic of Science Fiction", Science Fiction Studies \#6 Vol 2.2, July 1975. http:// www.depauw.edu/sfs/backissues/6/russ6art.htm

Voir aussi Donna Spalding Andréolle, Dialectique idéologique et sociale dans la science-fiction féminine aux États-Unis, 1966-1996: Querelles du présent, utopies du futur, Presses universitaires du Septentrion, Villeneuve d'Ascq, 1999, p. 244-245.

13. Pour un développement de ces idées, voir Donna Spalding Andréolle, «"Beam Me Up Scotty, There's No Intelligent Life Down Here": The Failed (Counter)cultural Message of Star Trek the Original Series (1966-1969) », EOLLE n¹, The Woodstock Years, U. Le Havre, décembre 2011, pages 39-46, https://gric.univ-lehavre.fr/spip.php?article124

14. Protestations qui poussèrent néanmoins la chaîne $\mathrm{CBS}$ à permettre la rediffusion de la série sous forme de franchise aux chaînes affiliées dans les années 1970.

15. Pour le développement plus complet de cette analyse, voir Donna Spalding Andréolle, «Echoes of the War on Terror and Post-911 America in Battlestar Galactica (2003-2009) » dans TV/ Series 4/2013, Écho et reprise dans les séries télévisées (II): Représentations - enjeux socio-culturels, politiques et idéologiques de la reprise, https://tvseries.revues.org/744

16. Voir aussi Lori Maguire, "Why Are We as a People Worth Saving?" Battlestar Galactica and the Global War on Terror », TV/Series [En ligne], 1 | 2012, URL : http://tvseries.revues.org/1519

17. Ronald D. Moore, Battlestar Galactica, The Series Bible, version pdf du manuscrit daté du 17 décembre 2003, source : http://www.harvardwood.org/resource/resmgr/hwp-pdfs/ battlestar_galactica_series.pdf (téléchargé le 26/8/2012).

18. Voir Ursula K. Le Guin, The Left Hand of Darkness, New York, Ace Books, 1969 ; Suzy McKee Charnas, Walk to the End of the World, New York, Del Rey, 1974, et Octavia E. Butler, Parable of the Sower, New York, Seven Stories Press, 1993.

19. Peur qui marqua notamment le passage à l'an 2000 avec les rumeurs qu'un virus « $Y 2 \mathrm{~K}$ » entraînerait une panne de tous les systèmes informatiques mondiaux.

20. ROSLIN. IT IS SIMPLy NOT ENOUGH TO KILL CYLONS, BECAUSE THEY DO NOT DIE. THEY RESURRECT THEMSELVES AND CONTINUE TO WALK AMONG US. IT IS HORRIFYING.

21. On pense ici à des séries comme 24 heures chrono (Fox, 2001-2008) plus particulièrement à partir de la saison 2), Rubicon (AMC, une seule saison en 2010), The Event (NBC, une seule saison en 2010-2011) et Homeland (Showtime, 2011-). De ces trois séries, The Event appartient au genre de la science-fiction et partage le traitement ambigu de l'altérité de Battlestar Galactica. La série met en scène des confrontations entre le président cubain-américain noir des États-Unis, qui souhaite libérer des extra-terrestres incarcérés depuis soixante-six ans dans une base militaire secrète en Alaska, et ses conseillers les plus proches qui redoutent un complot contre l'humanité venant de ces êtres qui ressemblent en tout aux humains. Le scénario révèle l'existence de deux « camps » parmi les aliens, certains désirant une vie paisible sur Terre, les autres voulant utiliser leurs pouvoirs supérieurs pour conquérir la Terre et anéantir l'espèce humaine. 


\section{RÉSUMÉS}

Cet article explore les articulations entre l'humain et le posthumain dans les deux versions de la série télévisée Battlestar Galactica, celle de Glen Larson (1978-1979) et le reboot de Ronald D. Moore (2003-2009). Au cœur de la saga - et en particulier dans sa version revisitée par Ronald D. Moore - se situe la figure du Cylon, être artificiel créé par l'homme mais dont l'évolution ne se réalise pleinement qu'après une rébellion contre l'espèce humaine. Alors que dans la série d'origine, les Cylons sont essentiellement une armée de robots commandée par le traître Baltar, on y discerne déjà un questionnement sur la frontière entre machine et humain. Ces questionnements sont au cœur de la version de 2004 : la transformation du Cylon en une créature physiquement ressemblante à l'homme, programmée dès lors à se comporter en humain, pose de réels problèmes tant aux colons en guerre qu'aux Cylons eux-mêmes. Au-delà de la réactualisation des débats sur l'humain, le posthumain et (plus récemment) sur le transhumain, cet article analyse les mises en relation entre les visions de l'altérité proposées dans les deux séries et les évolutions culturelles du genre science-fictionnel américain dans deux contextes sociopolitiques très contrastés.

This article explores the articulations between the concepts of human and post-human in the two versions of the TV series Battlestar Galactica, Glen Larson's (1978-79) and Ronald D. Moore's reboot, first aired in 2003. At the center of both versions, but more specifically in the more recent one, stands the figure of the Cylon, a man-made creation which only reaches its full potential after a rebellion against its creators. Although the Cylon in Glen Larson's version is simply an army of robot-soldiers under the command of the traitor Baltar, it is possible to perceive (in such characters as the Cylon Lucifer) a budding reflection on the blurred lines between man and machine. In Ronald D. Moore's version, however, this consideration is the driving force behind the development of the Cylon characters, since the Cylon is now a creature who resembles a human both physically and emotionally, a source of multiple moral issues for humans and cylons alike. Beyond the philosophical challenges of understanding the current debates on the human, the post-human and more recently the trans-human, this article seeks to analyze how Otherness is portrayed in both versions as well as the evolution of American science fiction in two different socio-political contexts.

\section{INDEX}

Mots-clés : Battlestar Galactica, science-fiction, posthumanité, guerre froide, Amérique post-9/11, post-11 septembre 2001, reboot, robot

Keywords : Battlestar Galactica, American science fiction, posthumanity, Cold War, post-9/11 culture, reboot, robot 


\section{AUTEUR}

\section{DONNA SPALDING ANDRÉOLLE}

Donna Spalding Andréolle est professeur des universités émérite en études américaines à l'Université Le Havre Normandie. Ses travaux de recherche sont consacrés aux articulations entre science et science-fiction, et aux aspects culturels des séries télévisées telles que Star Trek the Original Series, The Middle et Battlestar Galactica. Elle a co-édité deux recueils d'articles : Science and Empire in the $19^{\text {th }}$ Century: A Journey of Conquest and Progress avec Catherine Delmas et Christine Vandamme (Cambridge Scholars Publishing, 2010) et Women and Science, $17^{\text {th }}$ Century to Present: Pioneers, Activists and Protagonists avec Véronique Molinari (Cambridge Scholars Publishing, 2011), et a publié une monographie sur la série Friends, Friends: Destins de la Génération X (PUF, 2015). Donna Spalding Andréolle is professor emeritus of American Studies at University Le Havre Normandie (France). Her research focuses on the articulations between scientific discourse and science fiction as well as on the cultural aspects of TV shows like Star Trek (1966-69), The Middle and Battlestar Galactica. She is the co-editor of two collections, Science and Empire in the $19^{\text {th }}$ Century: A Journey of Conquest and Progress (with Catherine Delmas and Christine Vandamme, Cambridge Scholars Publishing, 2010) and Women and Science, $17^{\text {th }}$ Century to Present: Pioneers, Activists and Protagonists (with Véronique Molinari, Cambridge Scholars Publishing, 2011). She has most recently published a book in French on the series Friends (Friends: Destins de la Génération X, Presses Universitaires de France, 2015). 